\title{
Correction to: Initial time dependence of wind- and density-driven Lagrangian residual velocity in a tide-dominated bay
}

\author{
Guangliang Liu ${ }^{1,2} \cdot$ Zhe Liu $^{2,3}$ (D) Huiwang Gao ${ }^{2} \cdot$ Shizuo Feng ${ }^{2}$
}

Published online: 10 April 2021

(C) Springer-Verlag GmbH Germany, part of Springer Nature 2021

\section{Correction to: Ocean Dynamics (2021) 71:447-469} https://doi.org/10.1007/s10236-021-01447-y

The Publisher regrets that the first sentence of the Abstract was unfortunately printed incorrectly in the original version of this article. It should read "The nonlinear effect of the summer southeast wind and density on the 3D structures of the full Lagrangian residual velocity (LRV) was quantified for a generally nonlinear system, using Jiaozhou Bay (JZB), China as the test site".

The original article has been corrected.

The online version of the original article can be found at https://doi.org/ 10.1007/s10236-021-01447-y

\section{Zhe Liu}

zliu@ouc.edu.cn

1 Shandong Computer Science Center (National Supercomputer Center in Jinan), Shandong Provincial Key Laboratory of Computer Networks, Qilu University of Technology (Shandong Academy of Sciences), Jinan 250101, China

2 Key Laboratory of Marine Environment and Ecology, Ministry of Education, Ocean University of China, Qingdao 266100, China

3 Present address: Department of Earth Sciences, National Natural Science Foundation of China, Beijing, China 